\title{
Tres Décadas de Desigualdad y Erosión de la Clase Media en México
}

\author{
Three Decades of Inequality and ERosion of the Middle Class in Mexico
}

\author{
Genaro Aguilar Gutiérrez (*)
}

Resumen: El número de pobres en México aumentó en 7.8 millones de personas entre los años 1984 a 2010. Este artículo muestra con cálculos a partir de micro datos de la Encuesta Nacional de Ingresos y Gastos de los Hogares de México; la evolución de la desigualdad; de la pobreza y de la magnitud de la llamada clase media. Se estimaron las medidas de desigualdad (Gini, Theil, y razones de ingreso) y de pobreza (Proporción de pobres, índices de Sen y de Foster, Greer y Thorbecke); y con una metodología estandarizada a nivel mundial propuesta por Esteban y Ray (2007); se calculó la magnitud de la clase media en México y se cuantificaron las medidas de polarización para las clases sociales por ingreso familiar per capita. La principal conclusión es que el modelo económico adoptado en México a partir de la década de 1980 tuvo como resultado no sólo el aumento sistemático del número de personas en situación de pobreza y la desigualdad, sino también la erosión sistemática de la llamada clase media. La tendencia a una creciente desigualdad parece persistente en el largo plazo en México teniendo como corolario la disminución del mercado interno, representado por la disminución persistente de la clase media mexicana.

Palabras clave: Distribución del ingreso. Clase media y vulnerabilidad en México.

Clasificación JEL: I30, D31, D63

\begin{abstract}
The number of poor in Mexico increased by 6.8 million people between 2005 and 2010. This article shows calculations using micro data from the National Household Income and Expenditure Survey of Mexico; the evolution of inequality, poverty and the size of the middle class. Were estimated inequality measures (Gini, Theil, and income ratios) and poverty (Proportion of poor rates Sen and Foster, Greer and Thorbecke indexes) and with a globally standardized methodology proposed by Esteban and Ray (2007), we calculated the size of the middle class in Mexico and quantified the polarization measurements with two groups of household income per capita. The main conclusion is that the economic model adopted in Mexico since the 1980s has resulted in not only the systematic increase in the number of people in poverty and inequality, but also the systematic erosion of the middle class. The trend towards increasing inequality seems persistent in the long run in Mexico having a concomitant decrease in the domestic market, represented by the persistent decline of the Mexican middle class.
\end{abstract}

Key words: Income distribution. Middle class, vulnerability in Mexico.

JEL: I30, D31, D63

(*) Doctor en Ciencias Económicas por la Universidade Estadual de Campinas, Sao Paulo, Brasil. Profesor Titular C en el Posgrado en Ciencias Económicas del Instituto Politécnico Nacional, México. Esta investigación es resultado del proyecto de investigación denominado "Mercados de Trabajo, Desigualdad y Pobreza en México y Brasil 2000-2010", proyecto con registro número 20121150 de la Secretaría de Investigación y Posgrado (SIP) del IPN. El autor actualmente es Posdoctorando en el Programa de Pós-graduação Interunidades em Integração da América Latina (PROLAM) en la Universidad de Sao Paulo, Brasil. El autor desea agradecer a los árbitros anónimos por la revisión y observaciones realizadas a una versión previa de este artículo.E-mail: <genaro68mx@yahoo.com.br>. Recebido em: 30.08.2012 e aceito em 15.10.2012. 


\section{INTRODUCCIÓN: TRES DÉCADAS DE UN MODELO ECONÓMICO EXCLUYENTE}

De acuerdo con diversos autores, en México se aplicó, a partir de la década de 1980, la estrategia económica neoliberal - basada en la liberalización del comercio exterior, en la desregularización y liberalización del sistema financiero, en la liberalización de la inversión extranjera, en la ortodoxia macroeconómica, en la severa reducción de las políticas de fomento al desarrollo económico y, en general, en la reducción de la participación del estado en la economía (Calva, 2010; Aguilar 2000 y Guillén, 1996). La aplicación de políticas económicas de este tipo fue resultado de la adopción del "Washington Consensus", decálogo de políticas económicas que comprende: i, ii y iii) liberalización del comercio exterior, del sistema financiero y de la inversión extranjera; iv) orientación de la economía hacia los mercados externos; v) privatización de las empresas públicas como fin en sí mismo (es decir, sin adoptar, en las áreas de interés público, las medidas precautorias y el marco regulatorio para asegurar su mejor funcionamiento y el de los respectivos mercados); vi) desregulación de las actividades económicas; vii) la estricta disciplina fiscal (mecanismo que elimina el papel activo de la política fiscal para regular el ciclo económico); viii) eliminación de los desequilibrios fiscales previos, pero no mediante una mayor recaudación tributaria sino a través de la reducción de la inversión y el gasto públicos (que conduce a la supresión o reducción de programas de fomento económico general y sectoriales); ix) una reforma fiscal claramente orientada a reducir las tasas de impuesto a los ingresos mayores, ampliando en contrapartida la base de contribuyentes; $\mathrm{y} x$ ) un adecuado marco legislativo e institucional para resguardar los derechos de propiedad (Calva: 2010; Williamson: 1990).

En México parte importante del ancla del modelo económico fueron los salarios, que en términos reales perdieron más de $43.1 \%$ de su poder de compra entre los años 1982 y 2012.

El resultado de la aplicación de tres décadas de un modelo económico liberal fue que México perdió la carrera por el desarrollo económico. La clase media poco a poco fue reduciéndose. Si lo comparamos con Corea del Sur, por ejemplo, México tenía en 1982 un PIB per capita de 11,668 dólares (a dólares constantes del año 2009) mientras que Corea no tenía siquiera la mitad de eso, con 6,676 dólares. Así, en 1982 el PIB per capita mexicano era casi el doble del de Corea del Sur. En el año 2010 el PIB per capita alcanzó en México apenas los 13,798 dólares mientras que en Corea del Sur dicho indicador fue de 28,430 dólares. En consecuencia, Corea cruzó la línea que separa a los países subdesarrollados de los industrializados o de altos ingresos mientras que México se quedó hasta abajó, estancado en el subdesarrollo que ahora parece ser crónico.

Comparado con los países con los cuales realizó su integración económica, México también quedó a la zaga. Durante el período 1983-2010 el PIB per capita de Estados Unidos creció a una tasa promedio anual de 1.8\%; Canadá creció a una tasa de 1.6\% pero México creció sólo a una tasa de $0.5 \%$ anual en todo ese período. Adicionalmente, la desigualdad en la distribución del ingreso aumentó drásticamente en México, teniendo como corolario un país con menos crecimiento económico, mayor desigualdad social y mayor pobreza. 
En suma México adoptó un modelo de desarrollo económico, a partir de la década de 1980, cuyos resultados fueron - como se mostrará en este artículo - el aumento de la desigualdad y la polarización del desarrollo. En entidades fundamentalmente urbanas ese fenómeno se ha traducido en incrementos notables de la población en situación de pobreza.

Este artículo se propone ilustrar, a partir de cálculos de datos que provienen de encuestas en hogares, la evolución de la desigualdad y de la clase media en México. La segunda sección, después de este apartado muestra los indicadores de desigualdad en México para dos cortes lógicos: 1984 a 2010 y 2005 a 2010; incluyendo un análisis de los cambios ocurridos en esos períodos y la evolución de la pobreza. La tercera parte muestra los resultados del cálculo de polarización del ingreso; exponiendo que en México la clase media perdió un importante espacio en la sociedad entre los años 1984 y 2010. Por último, se presentan las principales conclusiones del estudio.

\section{Evolución de la desigualdad y pobreza en MéXico}

Los datos disponibles de las Encuestas Nacionales de Ingresos y Gastos de los Hogares $^{(1)}$ muestran que entre los años 1984 y 2010 hubo un incremento en la desigualdad de la distribución del ingreso en México. Por ello aquí definimos ese proceso como "tres décadas de desigualdad en México". Así, el coeficiente de Gini ${ }^{(2)}$, medida clásica, muestra que la distribución del ingreso familiar per capita empeoró al menos en $21.2 \%$; al pasar en ese período de 0.493 en 1984 a 0.598 en el año 2010; el 10\% de los hogares más ricos que en el año 1984 concentraban $34.7 \%$ de la riqueza, aumentaron su participación a $52.1 \%$ en el año 2010; mientras que el número de pobres aumentó en al menos 7.7 millones de personas en esos años.

El cuadro 1 muestra la distribución del ingreso familiar per capita en México en el año 1984 obtenida a partir del procesamiento de los micro datos de la Encuesta Nacional de Ingresos y Gastos de los Hogares ${ }^{(3)}$. La primera columna contiene a los diferentes

(1) La "Encuesta Nacional de Ingresos y Gastos de los Hogares" (ENIGH) de México es similar a la "Pesquisa Nacional por Amostra de Domicílios" de Brasil. La ENIGH está es realizada cada dos años (desde 1990) por el Instituto Nacional de Estadística y Geografía (INEGI) de México.

(2) El coeficiente de Gini es una medida ampliamente empleada para analizar la distribución del ingreso debido a que cumple estrictamente cuatro propiedades que, de acuerdo con Cowell (1995:55-60), hacen de ella una buena medida de desigualdad: a) satisface el principio de transferencia, también conocido como condición Pigou-Dalton; b) es independiente de la escala con que se mida el ingreso de las personas (el valor de la medida no depende de la unidad monetaria del ingreso); c) satisface el principio de población (que establece que la medida deberá ser independiente del tamaño de la población analizada); y d) se puede descomponer.

(3) Nota metodológica: Las bases de micro datos originales de las Encuestas Nacionales de Ingresos y Gastos de los Hogares contenían información de ingreso y características de las familias en archivos por separado. En los archivos de ingreso de las personas los datos estaban presentados de tal forma que cada tipo de ingreso ocupaba un registro; de esa manera, para cada individuo había tantos registros como fuentes de ingresos tuviera: una persona podría tener 3 ó 4 registros en caso de que tuviera, respectivamente, 3 ó 4 fuentes de ingreso. Los archivos de características de las viviendas presentaban información relevante de las familias, como el número de integrantes, el factor de expansión y características socio-económicas del hogar. Todos los archivos distinguían a las personas y familias por folios o códigos clave. La forma de proceder para preparar los archivos necesarios para analizar la distribución del ingreso familiar per capita en el Estado de México fue, grosso modo, la siguiente. A) A partir de los archivos de ingreso se calculó el ingreso corriente 
estratos de la población ordenados por valores descendientes de ingreso. Así, el 1\% superior se refiere al 1\% de la población mexicana con ingreso más elevado; el 5\% comprende al sector de la población que equivale al $5 \%$ del total de los mexicanos pero que son los que mayor ingreso poseen; incluyendo al primer $1 \%$. A continuación observamos la distribución del ingreso familiar per capita por décimos de la población, comenzando también por el $10 \%$ más rico y terminando con el $10 \%$ más pobre de la sociedad.

En el cuadro 1 también se puede observar el porcentaje del ingreso nacional que tiene cada uno de los diferentes sectores de la sociedad mexicana. La segunda columna muestra los porcentajes de ingreso correspondiente a cada grupo. La tercera y cuarta columna permiten observar, respectivamente, valores acumulados desde los ingresos más altos hasta los más pequeños y viceversa. La quinta columna tiene información relativa al ingreso promedio en dólares, de cada estrato social. Finalmente, las dos últimas columnas presentan los ingresos relativos de cada grupo, en relación a la mediana del ingreso (columna 6) y en relación al ingreso promedio del estado (columna 7). En la parte inferior del cuadro están las medidas de desigualdad de Gini, Theil; así como la mediana del ingreso y el ingreso promedio en dólares de ese año.

Podemos observar que el subgrupo de la población mexicana más rica (el 1\% más rico) retenía, en 1984, el 16.7\% del ingreso y poseía un ingreso promedio de 3,276 dólares per capita mensual. Considerando el $5 \%$ más rico de la población, la participación relativa era de $31.05 \%$ y el ingreso promedio mensual de 704 dólares por persona. El "primer" décimo de la distribución (algunos autores denominan "primer decil a los más pobres" aquí denominaremos "último decil" o "último décimo" a los más pobres), el sector compuesto por el 10\% más rico de la población detentaba, en 1984, el 34.7\% del ingreso total; mientras que su ingreso promedio era de 394 dólares por persona en cada familia de ese estrato social. En el décimo siguiente se observa una caída abrupta en la participación ya que ese sector de la población solo poseía el 20.5\% del ingreso con ingreso promedio de 265.6 dólares por persona. Después de ese décimo de la población, las reducciones en las participaciones de los diferentes grupos son menores, sugiriendo una mayor desigualdad entre los ricos que entre los pobres de México.

Es importante notar la magnitud de la diferencia que existe entre la mediana del ingreso nacional (que es de 125 dólares) y el ingreso promedio (aproximadamente 196 dólares). Estos datos son relevantes porque muestran lo que técnicamente se conoce como una fuerte asimetría positiva en la distribución del ingreso en México, lo que es sinónimo de elevada desigualdad: mientras más se aleje la mediana del ingreso del ingreso promedio, mayor será la desigualdad en la distribución del mismo. Por ello, la razón de Kuznets muestra que el ingreso relativo del 1\% superior varía de 26.3 a 16.7 según sea hecha la comparación en relación a la mediana del ingreso o en relación al ingreso

total del individuo y se sumaron los ingresos de todos los individuos de cada familia, generando un nuevo archivo que contenía la información de ingreso total de cada familia; B) se cruzó este archivo (a través del folio) con el de las características de las familias para obtener, asociado a cada registro de ingreso familiar, el número de integrantes de la familia; C) en seguida, se calculó el ingreso familiar per capita; D) A partir de este ultimo tipo de archivos (para cada año) se calcularon todas las medidas de desigualdad y pobreza a que nos referiremos en esta primera fase del proyecto. 
promedio. El segmento compuesto por el $70 \%$ más pobre de la sociedad mexicana tenía percepciones inferiores al ingreso promedio.

Cuadro 1. Distribución del ingreso familiar per capita en México, 1984

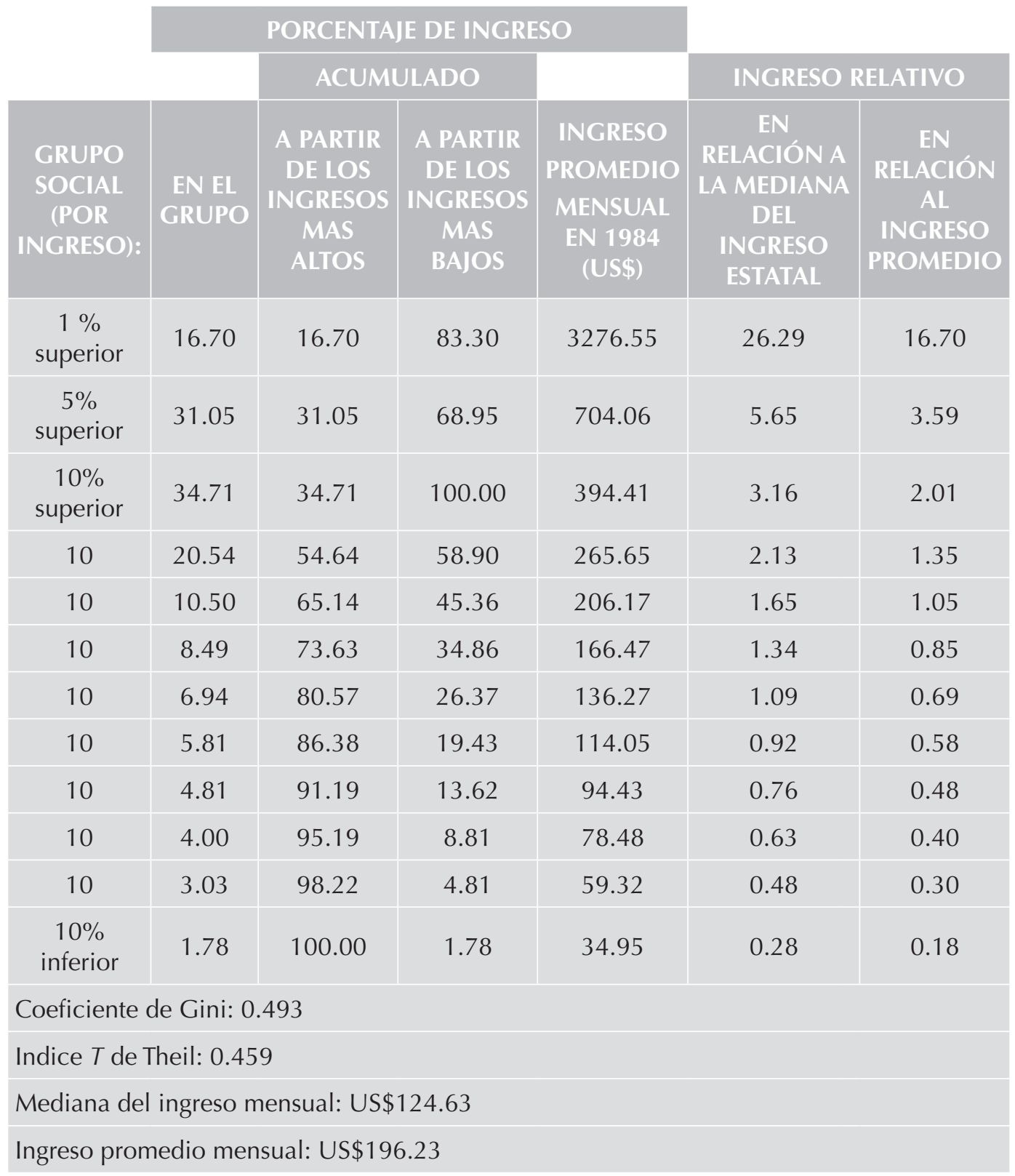

Fuente: Elaborado a partir del procesamiento de los microdatos de la "Encuesta Nacional de Ingresos y Gastos de los Hogares 1984". 


\section{1. ¿COMO SE MODIFICÓ ESTA SITUACIÓN EN CASI TRES DÉCADAS?}

El cuadro 2 muestra los resultados del cálculo de los percentiles de la distribución del ingreso familiar per capita en México pero ahora ya con datos al año 2010. Se puede observar que el ingreso del sector más adinerado de la sociedad mexicana (el 1\% más rico) aumentó como proporción del ingreso total a $36.1 \%$ (era de $16.7 \%$ ); mientras que el porcentaje del ingreso que retuvo el $5 \%$ más rico fue de $45.1 \%$ y el porcentaje del primer décimo de la sociedad pasó a ser el $52.1 \%$ del total. En el otro extremo, la participación en el ingreso total del último decil fue de 2.7\% (era de 1.78\%). Estos resultados muestran un fuerte proceso de polarización en la distribución del ingreso entre los años 1984 y 2010. Las medidas técnicas muestran que la desigualdad distributiva en México aumentó significativamente entre los años 1984 y 2010.

Cuadro 2. Distribución del ingreso familiar per capita en el Estado de México, 2010

\begin{tabular}{|c|c|c|c|c|c|c|}
\hline & & PORCENTA & JE DE INGRE & SO & & \\
\hline & & ACUMI & ULADO & & INGRESO R & RELATIVO \\
\hline $\begin{array}{c}\text { GRUPO } \\
\text { SOCIAL } \\
\text { (POR } \\
\text { INGRESO): }\end{array}$ & $\begin{array}{c}\text { EN EL } \\
\text { GRUPO }\end{array}$ & $\begin{array}{c}\text { A PARTIR } \\
\text { DE LOS } \\
\text { INGRESOS } \\
\text { MAS } \\
\text { ALTOS }\end{array}$ & $\begin{array}{c}\text { A PARTIR } \\
\text { DE LOS } \\
\text { INGRESOS } \\
\text { MAS } \\
\text { BAJOS }\end{array}$ & $\begin{array}{l}\text { INGRESO } \\
\text { PROMEDIO } \\
\text { MENSUAL } \\
\text { EN 2010 } \\
\text { (US\$) }\end{array}$ & $\begin{array}{c}\text { EN } \\
\text { RELACIÓN A } \\
\text { LA MEDIANA } \\
\text { DEL } \\
\text { INGRESO } \\
\text { ESTATAL }\end{array}$ & $\begin{array}{c}\text { EN } \\
\text { RELACIÓN } \\
\text { AL } \\
\text { INGRESO } \\
\text { PROMEDIO }\end{array}$ \\
\hline $\begin{array}{c}1 \% \\
\text { superior }\end{array}$ & 36.11 & 36.11 & 63.89 & 4751.00 & 31.00 & 20.21 \\
\hline $\begin{array}{c}5 \% \\
\text { superior }\end{array}$ & 45.12 & 45.12 & 54.88 & 1147.62 & 7.49 & 4.88 \\
\hline $\begin{array}{c}10 \% \\
\text { superior }\end{array}$ & 52.11 & 52.11 & 100.00 & 926.86 & 6.05 & 3.94 \\
\hline 10 & 8.53 & 57.11 & 53.89 & 321.44 & 2.10 & 1.37 \\
\hline 10 & 8.03 & 65.99 & 42.89 & 272.14 & 1.78 & 1.16 \\
\hline 10 & 7.64 & 74.44 & 34.01 & 204.76 & 1.34 & 0.87 \\
\hline 10 & 6.13 & 81.66 & 25.56 & 173.52 & 1.13 & 0.74 \\
\hline 10 & 4.72 & 87.46 & 18.34 & 146.36 & 0.96 & 0.62 \\
\hline 10 & 3.73 & 91.83 & 12.54 & 122.13 & 0.80 & 0.52 \\
\hline 10 & 3.36 & 95.14 & 8.17 & 102.29 & 0.67 & 0.44 \\
\hline 10 & 3.08 & 97.32 & 4.86 & 77.91 & 0.51 & 0.33 \\
\hline $\begin{array}{c}10 \% \\
\text { inferior }\end{array}$ & 2.68 & 100.00 & 2.68 & 46.25 & 0.30 & 0.20 \\
\hline Coeficiente & le Gini: 0 & 598 & & & & \\
\hline Indice $T$ de & heil: 0.52 & & & & & \\
\hline Mediana de & ingreso $\mathrm{n}$ & ensual: US & 53.24 & & & \\
\hline Ingreso pro & dio me & ual: US\$2 & & & & \\
\hline
\end{tabular}

Fuente: Elaborado a partir del procesamiento de los microdatos de la "Encuesta Nacional de Ingresos y Gastos de los Hogares 2010". 
Como se sabe, el índice de Gini es la medida clásica de desigualdad puesto que cumple con el principio Pigou-Dalton (aumentar ante cambios regresivos en la distribución del ingreso). Pues bien, el índice de Gini muestra que la desigualdad en la distribución del ingreso en México aumentó $21.3 \%$ en casi tres décadas; el índice de Theil sugiere un aumento $14.4 \%$. Técnicamente, la diferencia de incremento en estas dos medidas de desigualdad debe ser interpretada como un aumento generalizado en la desigualdad; con efectos importantes para la caída de la clase media: al caer un sector importante de dicho grupo social en situación de pobreza, el indice de Gini crece más rápido que el índice de Theil. Esto sugiere, como una de nuestras primeras conclusiones, que de aqui en adelante las politicas públicas a desarrollarse en México deberían enfatizar la atención de los grupos sociales que antes formaban parte de la clase media y que ahora están en situación de pobreza.

Se puede deducir que ese segmento de la población posee capital humano acumulado, capacidades acumuladas, que tienen patrimonio acumulado, pero que su ingreso corriente; como resultado de tres décadas de un modelo económico excluyente ha disminuido: se trata de profesionistas liberales (contadores, abogados, médicos; etc) cuyos despachos, oficinas, o consultorios han debido cerrar actividades en las zonas urbanas debido tanto al modelo económico como a la crisis económica que ha mermado el ingreso de sus principales clientes; pero cuya situación de pobreza moderada puede ser atendida a través de programas sociales de emergencia, temporales, y que podrían tener un efecto de resarcimiento de la situación anterior. Desde luego, de no atender a ese grupo de la sociedad, la situación de pobreza moderada que ahora viven podría perpetuarse y engrosar las filas de los pobres crónicos.

De estos hallazgos surge una pregunta relevante: Si ambos extremos de la sociedad mexicana mejoraron su posición relativa en la distribución del ingreso nacional, ¿qué transformación significativa ocurrió entonces en el perfil distributivo del ingreso?

Los cuadros 1 y 2 , leídos conjuntamente, arrojan luz sobre las modificaciones sustanciales que ocurrieron en la sociedad mexicana en las últimas tres décadas. Podemos constatar que el ingreso conjunto de los décimos $3^{\circ}$ a $7^{\circ}$ de la distribución del ingreso era, en el año 1984, de 36.6\% del ingreso total. Ya en el año 2010 dicha participación cayó a 30.3\%, lo que significó una pérdida 6.3 puntos porcentuales. En particular, el tercer décimo redujo su participación en 2.5 puntos porcentuales del ingreso (lo que significa que miles de millones de pesos pasaron de este sector de la población hacia otro). En otras palabras, quienes socialmente perdieron más en la participación en la distribución del ingreso en la sociedad mexicana en las últimas tres décadas fueron los deciles 4 a 8, que componen la llamada clase media emergente. Algunos autores sugieren que es el segundo décimo de la distribución donde se ubica la clase media: pues bien, ese sector de la población pasó de tener 20.5\% del ingreso en el año 1984 a poseer sólo el $8.5 \%$ de la riqueza en el año 2010; una constatación más de que la clase media mexicana se está empobreciendo.

Por lo tanto, es plausible considerar que un segmento importante de la población que anteriormente se encontraba en los sectores más vulnerables de la clase media mexicana (deciles 4, 5 y tal vez 6), pasaron, como resultado de la crisis económica mundial y del alza en los precios de los alimentos, a formar parte de la sociedad pobre. 


\subsection{EVOLUCiÓN RECIENTE DE LA POBREZA y LA DESIGUALDAD EN MÉXICO}

El cuadro número 3 presenta los resultados, para los años 2005 y 2010, de las medidas de pobreza empleadas por el CONEVAL ${ }^{(4)}$ (pobreza alimentaria, de capacidades y de patrimonio) ${ }^{(5)}$, así como las medidas de desigualdad (índices de Gini, Theil, Atkinson y Razón de Kuznets) y de pobreza clásicas (índice de pobreza de Sen, índice de pobreza de Foster-Greer y Thorbecke conocida en la literatura especializada como FGT y la razón de insuficiencia de ingresos); para toda la república mexicana y para los estados de México, Jalisco, Michoacán, Guanajuato y Chiapas, además del Distrito Federal. Siguiendo la metodología ya indicada anteriormente, los datos que sirvieron de base para estos cálculos provienen de la Encuesta Nacional de Ingresos y Gastos de los Hogares para los años 2005 y 2010; se tomaron los datos uno a uno de los integrantes de los hogares entrevistados en el marco de dicha encuesta, es decir se tomaron los microdatos para realizar todos los cálculos de los índices de desigualdad (Gini, Theil, etc.) y de pobreza (Sen, FGT, etc.).

Los resultados presentados en el cuadro 3 muestra que la pobreza alimentaria pasó de $19.4 \%$ (en 2005) a 23.8\% de la población mexicana en 2010. De igual manera, la pobreza de capacidades pasó de constituir 26.3\% de la población en 2005 a 33.5\% en 2010 . Por último, la pobreza patrimonial que abarcaba a 48.2\% de los mexicanos en 2005 pasó a $51.6 \%$ de la población en el año 2010 .

Así, el elemento que llama la atención es que en todo el país se observa un aumento sistemático en la desigualdad y de la pobreza. En términos absolutos el número de pobres en México subió de 50.14 millones en 2005 a 57.85 millones en 2010; un aumento de $15.4 \%$ ! Pero lo que más creció fue la pobreza alimentaria. Efectivamente, la población mexicana en esta situación pasó de 20.2 a 26.7 millones de mexicanos; un aumento de $32.07 \%$ !

Por entidades del país destaca el aumento de la pobreza absoluta es como sigue: estado de México, 13.3\%; DF 15.2\%; Jalisco, 11.5\%; Michoacán, 6.35\%; Guanajuato $11.1 \%$ y destaca Chiapas, donde la pobreza creció 16.8\%. Debemos aquí hacer notar que el número de pobres alimentarios en el Distrito Federal creció 51\% mientras que en Chiapas aumentó, 46.09\%; sin embargo el número absoluto de pobres en Chiapas es 4 veces superior al del DF.

(4) El CONEVAL (Consejo Nacional de Evaluación de la Política de Desarrollo Social), instaurado en el año 2006; es el organismo oficial de cálculo de la pobreza en México.

(5) El CONEVAL de México calcula niveles de pobreza en México a partir de los ingresos per capita con las siguientes definiciones: pobreza alimentaria: incapacidad para obtener una canasta básica alimentaria, aun si se hiciera uso de todo el ingreso disponible en el hogar para comprar sólo los bienes de dicha canasta; pobreza de capacidades: insuficiencia del ingreso disponible para adquirir el valor de la canasta alimentaria y efectuar los gastos necesarios en salud y en educación, aun dedicando el ingreso total de los hogares nada más para estos fines; pobreza de patrimonio: insuficiencia del ingreso disponible para adquirir la canasta alimentaria, así como para realizar los gastos necesarios en salud, vestido, vivienda, transporte y educación, aunque la totalidad del ingreso del hogar sea utilizado exclusivamente para la adquisición de estos bienes y servicios. Por lo tanto, quienes tienen pobreza alimentaria no pueden alimentarse con su ingreso. Quienes tienen pobreza de capacidades pueden no tener pobreza alimentaria, pero no tienen ingreso para ejercer (además de alimentos) gastos en salud y educación. Ya los pobres de patrimonio pueden no tener pobreza alimentaria ni de capacidades, pero no tienen ingreso suficiente para adquirir vestido, vivienda y transporte. 
Algunos aumentos en las medidas clásicas de pobreza destacan: aumentó 5.8\% la insuficiencia de ingresos a nivel nacional y 30.3\% en el Distrito Federal (los pobres son más pobres ahí). En todos los estados crece la desigualdad: medido por el índice de Gini los mayores incrementos se registran en el estado de México y Guanajuato. El índice de Theil creció 40\% en el Distrito Federal (ello significa que las diferencias entre los ricos aumentaron, se concentró más la riqueza). La razón de Kuzntes, que mide el ingreso de los más ricos como proporción del ingreso de los más pobres (ingreso del primer decil entre ingreso de los 4 últimos deciles) aumentó significativamente en los estados de México y Michoacán, con $62.7 \%$ y $60.7 \%$.

Estos resultados sugieren varias conclusiones parciales: a) la pobreza en esos estados y a nivel de país, en México, creció entre 2005 y 2010; b) para todo México la pobreza alimentaria aumentó en mas de 6 millones de personas (32\% en sólo 5 años); c) la pobreza patrimonial aumentó en15.3; d) más de la mitad de la población mexicana (56.9\%) vive en algún tipo de pobreza; e) a nivel nacional todos los indicadores de pobreza (índice FGT, Sen e insuficiencia de ingresos) aumentan.

Las medidas de desigualdad muestran, tanto a nivel nacional como para los 6 estados ahí incluidos, un aumento sistemático de la desigualdad. 


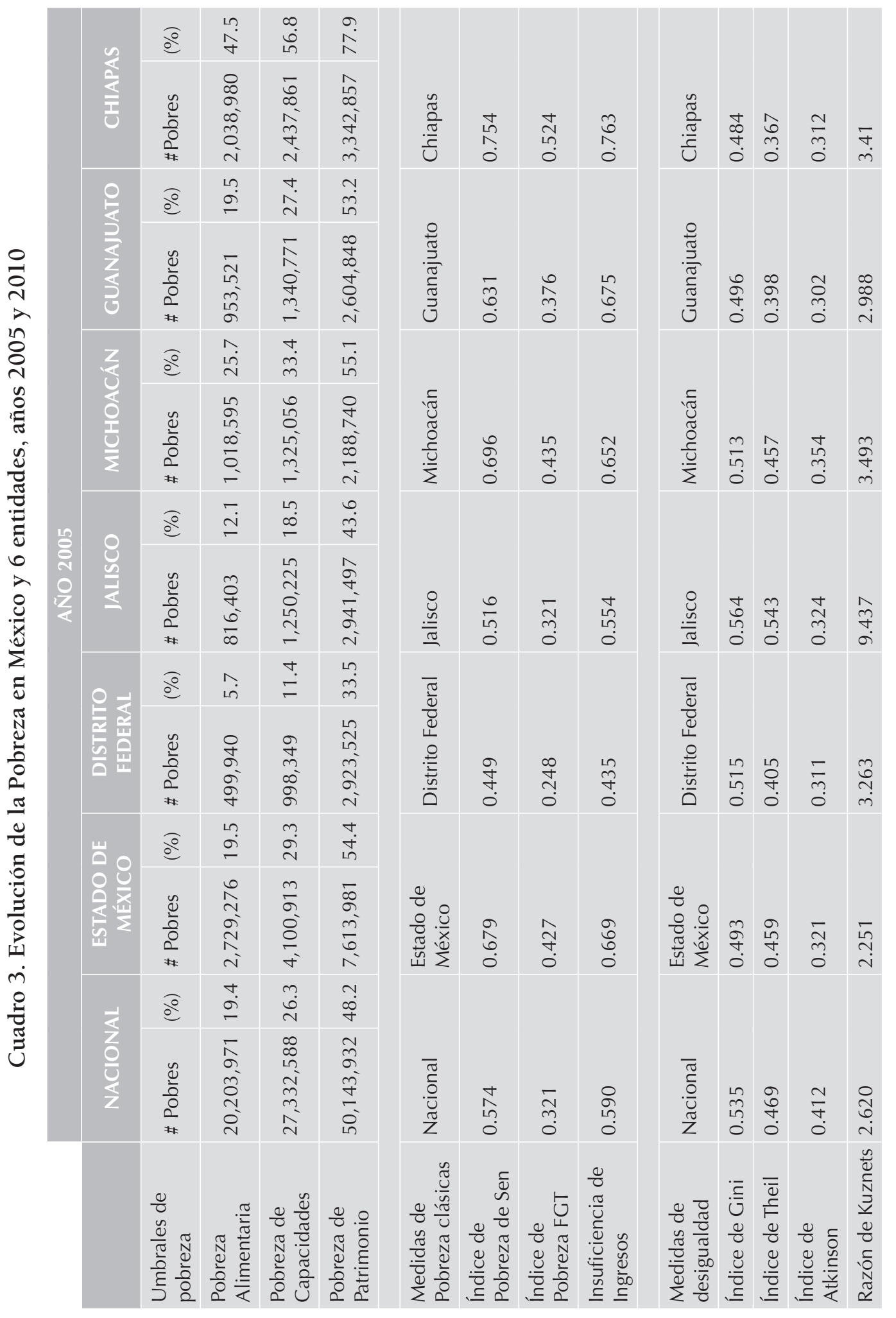




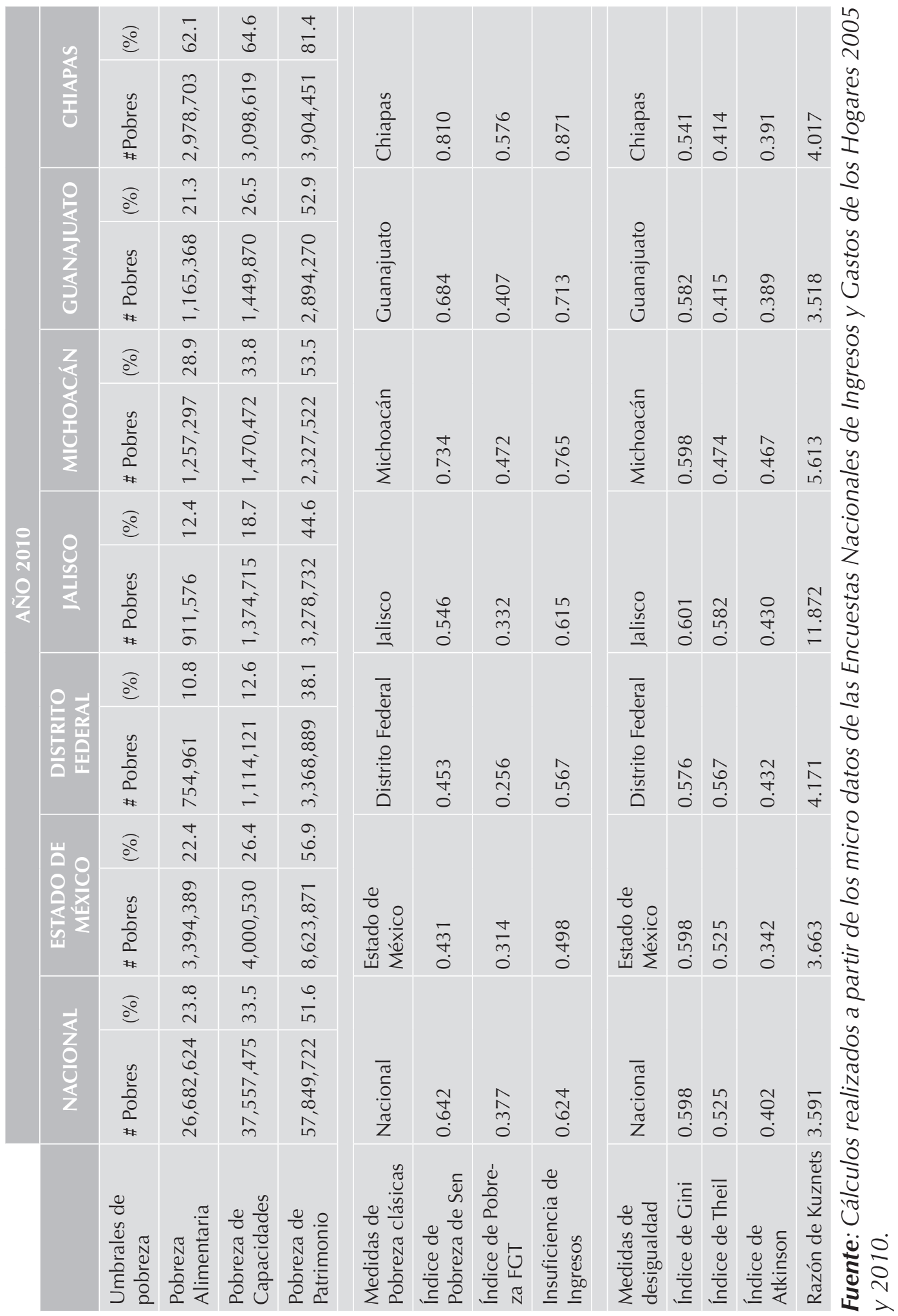




\section{3. ¿Cómo Medir la eVOluCión de la Clase Media?}

La metodología estandarizada a nivel internacional para cuantificar la evolución de la clase media consiste en la aplicación de un índice de polarización. El antecedente es que, a partir de la década de 1980, diversos trabajos analizaron el fenómeno de reducción de la clase media en Estados Unidos, con el crecimiento de los extremos de la distribución del ingreso (Kuttner, 1983; Thurow, 1984; Rosenthal, 1985, Davis y Huston, 1992). Wolfson (1994) y Esteban y Ray (1994) mostraron que la realización del análisis por medio de medidas de desigualdad tradicionales era insuficiente para la comprensión adecuada del fenómeno, haciendo necesaria la utilización de otros índices que permitieran captar apropiadamente la disminución de la clase media.

A grandes rasgos la idea es que al tratar a los individuos de un mismo grupo de forma homogénea, es decir, como si tuvieran el mismo ingreso, estamos cometiendo un grave error de aproximación. Obviamente, el error será menor cuanto mas parecidos sean los integrantes de una clase o grupo social entre sí.

La idea es deducir ese error, de modo que la medida de polarización propuesta por los autores está dada por:

$$
P(f, \alpha, \beta)=P(\pi, y)-\beta \varepsilon(1)
$$

- donde $P(\pi, y)$ es la medida de polarización desarrollada por Esteban y Ray $(1994)^{(6)}$ y $\varepsilon$ es el error de aproximación cometido. El parámetro $\beta$ mide el peso que atribuimos al error de medida y es seleccionado de forma ad oc por el investigador.

En una extensión muy reciente del índice de polarización original, Esteban, Gradín y Ray (2007) muestran que el error de aproximación es minimizado cuando seleccionamos valores de ingreso de tal forma que el ingreso que divide cualquiera de los intervalos adyacentes es igual al ingreso promedio de esos dos intervalos.

Así, el error de medida óptimo estaría dado por la diferencia entre el coeficiente de Gini observado y el calculado, asumiendo que todos los individuos de un grupo tengan el mismo ingreso. Es decir:

$$
\varepsilon=(G(f)-G(\rho)(2)
$$

- donde $G(f)$ representa el índice de Gini de la distribución del ingreso observado y G( $\rho)$ representa el valor del coeficiente de Gini en caso de que todos los individuos de un mismo grupo tengan el mismo ingreso. Combinando (1) y (2), llegamos a la medida de polarización ampliada propuesta por Esteban, Gradín y Ray (2007):

$$
P(f, \alpha, \beta)=P(\pi, y)-\beta[G(f)-G(\rho)]
$$

(6) Con base en una serie de axiomas, Esteban y Ray (1994) definieron una medida de polarización como:

$P(\pi, y)=\sum_{i=1}^{n} \sum_{j-1}^{n} \pi_{i}^{1+a}\left|\mu_{i}-\mu_{j}\right| \propto \in[1 ; 1.6]^{2}$

donde $\mathbb{I}_{i}$ y $\mathbb{I}_{j}$ representan la proporción de individuos en los grupos i y $j ; \mu_{i}$ y $\mu_{j}$ son los ingresos promedio de las personas en los grupos $i$ y $j$. El término $\left|\mu_{i}-\mu_{j}\right|$ capta la diferencia absoluta en los ingresos promedio entre los grupos $j$ e $i$. Así, cuanto mayor sea la distancia entre los ingresos de los dos grupos, mayor será la polarización. El parámetro $\alpha$ indica el peso dado al tamaño de los grupos en la medida de polarización. 
Resulta relevante observar que esta medida de polarización permite la minimización al máximo del error en el cálculo de medidas clásicas de desigualdad, como el índice de Gini; cuando los cálculos son realizados a partir de datos agrupados por clases de ingreso.

El término entre corchetes - desigualdad total menos desigualdad entre los grupos - mide la desigualdad dentro de los grupos. De este modo, cuanto mayor sea la dispersión dentro de cada grupo, menor será la polarización de una dada representación $\rho$.

Esta es la medida de polarización utilizada en este trabajo. La calculamos para la división de las familias mexicanas en dos y tres grupos sociales. En particular, un subproducto adicional del análisis de la división de las familias en tres grupos sociales es la obtención de los valores monetarios que definen las clases baja, media y alta de ingreso de forma no arbitraria.

En suma, inicialmente encontraremos los valores $y_{i}$, If y $\mu$, que definen la representación $\rho$ de la distribución del ingreso observada $f$. En seguida, obtendremos la desigualdad dentro de los grupos $[G(f)-G(\rho)]$ y, finalmente, calculamos el índice de polarización definida en (3).

\section{Resultados: polarización del ingReso y deCliVe de la Clase media en MéXiCo}

El cuadro 4 presenta el índice de polarización extendida, con $\beta=1$ y tres valores diferentes de $\alpha(1 ; 1.3 \text { y } 1.6)^{(7)}$. Un primer aspecto a ser destacado en el cuadro 4 es que la polarización del ingreso per capita es mayor que en los otros criterios de ingreso, y ocurre lo mismo con el índice de Gini. Así, en la medida en que damos menos ponderación o peso a las personas dentro de una misma familia, hay una reducción automática en los índices de desigualdad y de polarización. Ello se debe al hecho de que las familias con menor ingreso en México presentan sistemáticamente un mayor número de hijos, sean adultos o niños.

Cuadro 4. Índices de polarización extendida y de Gini en México, 1984 y 2010

\begin{tabular}{|c|c|c|c|c|c|c|c|c|c|c|c|c|}
\hline & \multicolumn{4}{|c|}{ IFP } & \multicolumn{4}{|c|}{ IFC1 } & \multicolumn{4}{|c|}{ IFC_OCDE } \\
\hline & $\alpha=1$ & $\alpha 1.3$ & $\alpha=1.6$ & Gini & $A=1$ & $\alpha=1.3$ & $\alpha=1.6$ & Gini & $\alpha=1$ & $\alpha=1.3$ & $A=1.6$ & Gini \\
\hline \multicolumn{13}{|c|}{2 GRUPOS } \\
\hline 1984 & 0.278 & 0.214 & 0.165 & 0.520 & 0.263 & 0.202 & 0.155 & 0.500 & 0.264 & 0.203 & 0.155 & 0.501 \\
\hline 2010 & 0.290 & 0.224 & 0.173 & 0.580 & 0.267 & 0.204 & 0.155 & 0.547 & 0.266 & 0.203 & 0.154 & 0.547 \\
\hline
\end{tabular}

(7) Trabajamos con tres diferentes niveles de ingreso familiar: el ingreso familiar per capita, al que denominaremos IFP; y, siguiendo la metodología de Esteban, Gradín y Ray (2007); el ingreso familiar ajustado mediante dos diferentes escalas de equivalencia. En la primera, dividimos el ingreso familiar entre la raíz cuadrada del número de personas de la familia, la cuál llamaremos IFC1; en la segunda, utilizamos ponderaciones para adultos y niños, de acuerdo con la escala de equivalencia de la Organización para la Cooperación y el Desarrollo Económico (OCDE), a la cuál llamamos IFC_OCDE. 


\begin{tabular}{|l|l|l|l|l|l|l|l|l|l|l|l|l|}
\hline \multicolumn{10}{|c|}{3 GRUPOS } \\
\hline 1984 & 0.254 & 0.172 & 0.114 & 0.520 & 0.249 & 0.171 & 0.115 & 0.500 & 0.249 & 0.171 & 0.116 & 0.502 \\
\hline 2010 & 0.281 & 0.201 & 0.143 & 0.580 & 0.262 & 0.185 & 0.130 & 0.547 & 0.267 & 0.184 & 0.128 & 0.547 \\
\hline
\end{tabular}

Fuente: Cálculos propios a partir de los micro datos de la Encuesta Nacional de Ingreso y Gasto de los Hogares de México, 1984 y 2010.

Al comparar los resultados del cálculo de los índices de polarización de 1984 y 2010 para $n=2$, podemos constatar un aumento, de alrededor de $5 \%$ para el ingreso familiar per capita (IFP), mientras que IFCl y IFC_OCDE se mantienen prácticamente estables en todo el período; mientras que los niveles de desigualdad medidos por el índice de Gini crecieron alrededor de $10 \%$.

Es decir, cuando se divide a la sociedad mexicana en solo dos grupos sociales, los resultados indicarían que el incremento en los niveles de desigualdad son mayores que los incrementos en el grado de polarización del ingreso. Esto, sin duda es un sesgo más bien estadístico ya que es mucho mejor, en una sociedad capitalista, considerar al menos la existencia de tres clases sociales, y no sólo de dos, como se verá a continuación.

Para el ejercicio con tres grupos o clases sociales, podemos observar nítidamente un aumento de la polarización del ingreso con todos los valores de $\alpha$; desde aumentos de $10 \%$ tomando el ingreso familiar per capita con $\alpha=1$; hasta un sorprendente aumento de $25.6 \%$ con $\alpha=1.6$. Por lo tanto, al considerar la división de la sociedad mexicana en tres clases sociales, la polarización del ingreso aumentó proporcionalmente más que el coeficiente de Gini entre los años 1984 y 2010.

Los estudios empíricos internacionales recientes, especialmente los realizados por los autores pioneros muestran que la división de la sociedad en tres grupos es congruente con el desarrollo del capitalismo en los países centrales, pero sobre todo en las naciones en vías de desarrollo.

Si comparamos los resultados para las familias mexicanas con los de los países de la OCDE calculados y reportados por Esteban, Gradín y Ray (2007), podemos observar que la polarización del ingreso en México es mucho mayor que en esos países ${ }^{(8)}$. Por ejemplo, analizando la representación tripolar de la IFC_OCDE para $\alpha=1$ en el período 1989-1992 (dependiendo del país), Esteban, Gradín y Ray (2007) encontraron: 0.157 para los Estados Unidos, 0.154 en el Reino Unido, 0.099 en Suecia, 0.111 en Alemania; y 0.128 en Canadá. A su vez, nuestros cálculos muestran que la polarización del ingreso en México en 1984 era ya de 0.249 y pasó a 0.267 en el año 2010.

En el cuadro 5 presentamos el ingreso promedio normalizado de los grupos o clases sociales que se analizan aquí, para el caso de México. Así, en cada año el valor del ingreso

(8) Esteban, Gradín y Ray (2007) utilizaron datos del Luxembourg Income Study (LIS), que proporciona datos de ingreso estandarizados para diversos países. El concepto de ingreso familiar per capita definido por el LIS es el ingreso del trabajo anual + ingreso de capital + ingreso de seguridad social + transferencias sociales — impuestos — contribuciones obligatorias, ajustados por las escalas de equivalencia de la OCDE. 
promedio de cada estrato social fue dividido por el ingreso promedio de todo el país del mismo año. La parte superior del cuadro 5 contiene información que evidencia la gran disparidad de ingreso entre estratos sociales. Mientras que el ingreso per capita promedio normalizado de los más pobres es de 0.3782 ; el ingreso del grupo más rico de la sociedad equivalía a 2.514 veces el ingreso global en 1984. Además de ello, los resultados indican que hubo una caída en el ingreso promedio en el grupo 1; es decir en el ingreso de los más pobres, en todos los índices. Por otro lado, se presentó un aumento en el ingreso promedio en el grupo de mayores ingresos entre 1984 y 2010 en todos los índices. Por ejemplo, entre 1984 y 2010 el ingreso de los más ricos pasó de equivaler a 2.514 veces el ingreso promedio global, a constituir 2.74 veces dicho ingreso. De esta forma, para el ingreso per capita ese movimiento ceteris paribus aumentó la polarización del ingreso social, ya que los dos grupos se alejaron. Esteban, Gradín y Ray (2010) observaron una tendencia similar para el caso de los Estados Unidos a partir de los años 80. Los autores mostraron que, entre 1974 y 1979, la distancia entre los grupos sociales disminuyó, pero volvió a crecer a partir de ese año.

\section{Cuadro 5. Ingreso promedio normalizado de las clases sociales en México,} 1984 y 2010

\begin{tabular}{|c|c|c|c|c|c|c|c|c|c|}
\hline & \multicolumn{2}{|c|}{$I F P$} & & \multicolumn{2}{|c|}{$I F C 1$} & & \multicolumn{2}{|c|}{ IFC_OCDE } & \\
\hline & $\begin{array}{c}\text { GRUPO } \\
1\end{array}$ & $\begin{array}{c}\text { GRUPO } \\
2\end{array}$ & & $\begin{array}{c}\text { GRUPO } \\
1\end{array}$ & $\begin{array}{c}\text { GRUPO } \\
2\end{array}$ & & $\begin{array}{c}\text { GRUPO } \\
1\end{array}$ & $\begin{array}{c}\text { GRUPO } \\
2\end{array}$ & \\
\hline 1984 & 0.3782 & 2.5140 & & 0.3106 & 4.0131 & & 0.3401 & 3.2926 & \\
\hline \multirow[t]{3}{*}{2010} & 0.3706 & 2.7403 & & 0.2951 & 4.4907 & & 0.3333 & 3.6548 & \\
\hline & \multicolumn{3}{|c|}{ IFP } & \multicolumn{3}{|c|}{ IFC1 } & \multicolumn{3}{|c|}{$I F C \_O C D E$} \\
\hline & $\begin{array}{c}\text { GRUPO } \\
1\end{array}$ & $\begin{array}{c}\text { GRUPO } \\
2\end{array}$ & $\begin{array}{c}\text { GRUPO } \\
3\end{array}$ & $\begin{array}{c}\text { GRUPO } \\
1\end{array}$ & $\begin{array}{c}\text { GRUPO } \\
2\end{array}$ & $\begin{array}{c}\text { GRUPO } \\
3\end{array}$ & $\begin{array}{c}\text { GRUPO } \\
1\end{array}$ & $\begin{array}{c}\text { GRUPO } \\
2\end{array}$ & $\begin{array}{c}\text { GRUPO } \\
3\end{array}$ \\
\hline 1984 & 0.2578 & 0.9975 & 3.8273 & 0.2982 & 1.0035 & 3.6217 & 0.2991 & 0.9967 & 3.6588 \\
\hline 2010 & 0.2742 & 0.8596 & 4.2066 & 0.2985 & 0.9159 & 3.7889 & 0.3029 & 0.9094 & 3.7718 \\
\hline
\end{tabular}

Fuente: Cálculos propios a partir de los micro datos de la Encuesta Nacional de Ingreso y Gasto de los Hogares de México

La parte inferior del cuadro 5 presenta los resultados de nuestros cálculos si dividimos a la sociedad en tres clases. Para todos los criterios de ingreso, el grupo intermedio que es el que representa a la clase media mexicana, fue en realidad el único que sufrió reducciones de ingreso en relación al promedio. Así, disminuyó la distancia entre el ingreso promedio de los grupos 1 y 2 y aumentó la referente a los grupos 2 y 3 .

Mientras que el ingreso per capita promedio de la clase media mexicana era 3.87 veces mayor que la del grupo de personas más pobres en 1984, la diferencia cayó a 3.13 en 2010. Por otro lado, el ingreso promedio de los más ricos pasó de ser 3.84 veces el del grupo 2 (clase media en 1984) a constituir 4.89 veces el ingreso promedio de la clase media en el año 2010; tendencias análogas se desprenden del cuadro 5 en las otras dos 
categorías de ingreso aquí estudiadas. Por lo tanto, es claro que se presentó un proceso de polarización en la distribución del ingreso en México, que condujo al deterioro gradual de la clase media en términos de ingreso relativo.

El cuadro 6 presenta el tamaño relativo de cada una de las clases sociales en México en los años 1984 y 2010. Para la división en dos grupos, hubo un sensible aumento del tamaño relativo del grupo de ingreso más bajo, lo que podría llevar a la conclusión de que se redujo la polarización. Sin embargo, la magnitud de ese aumento es muy pequeña, explicando así la modificación del índice de polarización mostrada en el cuadro 4. Un hecho interesante al comparar nuestros resultados, con los de Esteban, Gradín y Ray (2007); es que México era el país que tenía más familias en el grupo de ingreso menor (62.4\% en 1984), seguido de Alemania (61.0\%), Reino Unido (59.5\%), Canadá (58.5\%), Estados Unidos (57.5\%) y Suecia (53.6\%). Ello debería hacer que la polarización en México fuese menor que en los otros países, ceteris paribus, lo que no ocurre, debido a la gran diferencia entre los ingresos promedio de los grupos, ya mencionada.

Al realizar el análisis con tres clases sociales, el resultado conduce a la principal conclusión de esta investigación: se está "extinguiendo" la clase media en México. Mientras que el grupo de menor ingreso aumentó de tamaño en todo el período (los pobres pasaron de $56.6 \%$ a $63.9 \%$ de toda la población), el grupo 2, que constituye la clase media, disminuyó de $32.1 \%$ a $23.0 \%$ y el grupo 3, de los más ricos, aumentó de $11.3 \%$ a $13.1 \%$ de toda la población mexicana. Es decir, medida por el ingreso familiar per capita, la clase media constituía una tercera parte de la población en 1984; pero cayó a menos de una cuarta parte (23\%) en el año 2010.

Cuadro 6. Tamaño relativo de las clases sociales en México, 1984 y 2010 (en \%)

\begin{tabular}{|c|c|c|c|c|c|c|c|c|c|}
\hline & \multicolumn{2}{|c|}{ IFP } & & \multicolumn{2}{|c|}{ IFCI } & & \multicolumn{2}{|c|}{$I F C \_O C D E$} & \\
\hline & $\begin{array}{c}\text { GRUPO } \\
1\end{array}$ & $\begin{array}{c}\text { GRUPO } \\
2\end{array}$ & & $\begin{array}{c}\text { GRUPO } \\
1\end{array}$ & $\begin{array}{c}\text { GRUPO } \\
2\end{array}$ & & $\begin{array}{c}\text { GRUPO } \\
1\end{array}$ & $\begin{array}{c}\text { GRUPO } \\
2\end{array}$ & \\
\hline 1984 & 73.63 & 26.37 & & 72.33 & 27.67 & & 72.61 & 27.39 & \\
\hline \multirow[t]{3}{*}{2010} & 74.15 & 25.85 & & 73.30 & 26.70 & & 73.53 & 26.47 & \\
\hline & \multicolumn{3}{|c|}{$I F P$} & \multicolumn{3}{|c|}{$I F C 1$} & \multicolumn{3}{|c|}{$I F C_{-} O C D E$} \\
\hline & $\begin{array}{c}\text { GRUPO } \\
1\end{array}$ & $\begin{array}{c}\text { GRUPO } \\
2\end{array}$ & $\begin{array}{c}\text { GRUPO } \\
3\end{array}$ & $\begin{array}{c}\text { GRUPO } \\
1\end{array}$ & $\begin{array}{c}\text { GRUPO } \\
2\end{array}$ & $\begin{array}{c}\text { GRUPO } \\
3\end{array}$ & GRUPO & $\begin{array}{c}\text { GRUPO } \\
2\end{array}$ & $\begin{array}{c}\text { GRUPO } \\
3\end{array}$ \\
\hline 1984 & 56.61 & 32.06 & 11.33 & 59.14 & 28.75 & 12.11 & 59.31 & 28.52 & 12.17 \\
\hline 2010 & 63.93 & 23.01 & 13.06 & 62.43 & 24.42 & 13.15 & 62.42 & 24.50 & 13.09 \\
\hline
\end{tabular}

Fuente: Cálculos propios a partir de los microdatos de la Encuesta Nacional de Ingreso y Gasto de los Hogares de México.

\section{Conclusión ¿Está desapareciendo la Clase media en México?}

Una de las implicaciones del análisis de polarización del ingreso basada en la selección de la división de la sociedad en tres clases es que dicho análisis proporciona como 
subproducto los valores monetarios que dividen a los tres grupos sociales (pobres, clase media y ricos); es decir, los valores que minimizan la dispersión interna de las clases en relación a la dispersión de toda la distribución. Por lo tanto, podemos usar los grupos definidos por la metodología para trazar los límites no ad hoc entre las diferentes clases sociales. A continuación presentaremos los valores de ingreso que dividen a las clases sociales en México, medidos en valores reales del año 2010.

El primer punto que debemos indicar es que los límites de ingreso que resultan de nuestros cálculos llevan a que en la clase alta queden registradas únicamente las familias con un ingreso relativamente alto. Así, una familia de cuatro integrantes requiere un ingreso mensual de más de $\$ 121,728$ (es decir $\$ 30,432$ por persona en 2010 - unos 2,536 dólares) para pertenecer a la clase alta; para ser de los ricos. No obstante, la conclusión a la que se llegaría usando el sentido común es que en realidad esa familia forma parte de la clase media. Este hecho rebate el mito según el cual la clase alta en México, la de los ricos, está constituida exclusivamente por personas muy ricas, por familias multimillonarias. La verdad es que cerca de $11 \%$ de los hogares mexicanos pueden ser considerados clase alta. Un aspecto interesante que debe investigarse en el futuro y que justificaría la percepción equivocada de las personas en cuanto a la clase social a la que pertenecen es el hecho de que los ingresos no originados a partir del trabajo no son bien captados por las Encuestas Nacionales de Ingreso y Gasto de los Hogares. Se espera en realidad que haya una subestimación de los valores de ingreso, principalmente entre los más ricos, haciendo posible que el valor que divide a las clases sociales sea calculado, mediante nuestra metodología, en un valor mucho menor de lo que debería ser. Sin embargo, esta hipótesis es de difícil verificación.

\section{Cuadro 7. Valores de ingreso que dividían a las clases sociales en México, 1984 y 2010 (valores constantes del año 2010)}

\begin{tabular}{|c|c|c|c|c|c|c|}
\hline & \multicolumn{2}{|c|}{ IFP } & \multicolumn{2}{|c|}{$I F C 1$} & \multicolumn{2}{|c|}{ IFC_OCDE } \\
\hline & $\begin{array}{c}\text { POBRES- } \\
\text { CLASE } \\
\text { MEDIA }\end{array}$ & $\begin{array}{l}\text { CLASE } \\
\text { MEDIA- } \\
\text { RICOS }\end{array}$ & $\begin{array}{l}\text { POBRES- } \\
\text { CLASE } \\
\text { MEDIA }\end{array}$ & $\begin{array}{l}\text { CLASE } \\
\text { MEDIA- } \\
\text { RICOS }\end{array}$ & $\begin{array}{c}\text { POBRES- } \\
\text { CLASE } \\
\text { MEDIA }\end{array}$ & $\begin{array}{l}\text { CLASE } \\
\text { MEDIA- } \\
\text { RICOS }\end{array}$ \\
\hline 1984 & $\$ 8,646.24$ & $\$ 29,695.77$ & $\$ 16,511.87$ & $\$ 49,915.52$ & $\$ 12,161.36$ & $\$ 37,589.40$ \\
\hline 2010 & $\$ 9,517.62$ & $\$ 30,432.39$ & \$ $16,375.91$ & $\$ 50,547.36$ & $\$ 12,789.60$ & $\$ 39,825.64$ \\
\hline
\end{tabular}

Fuente: Cálculos propios a partir de la Encuesta Nacional de Ingreso y Gasto de los Hogares de México.

Un elemento que debe llamar la atención es que el ingreso necesario para pasar de la clase social más pobre a la clase media en México es extremadamente bajo (\$38,068 por familia; ó bien unos $\$ 9,517$ por persona al mes, unos 680 dólares) si lo comparamos con los estándares internacionales.

Los indicadores presentados en esta investigación muestran signos preocupantes del aumento de todas las dimensiones de la pobreza en México, como nación. Para fines de políticas públicas especialmente relevante es tener en mente la evolución de la pobreza alimentaria y la patrimonial. 
La pobreza alimentaria afecta a los más pobres entre los pobres. En ese sentido, experiencias internacionales recientes muestran algunas buenas prácticas en materia de políticas públicas y de transparencia en el acceso a los beneficios derivados de programas sociales; como en el caso de Brasil a partir del gobierno Lula. En México, a pesar de la propaganda oficial para promocionar y generar una imagen internacional favorable al Programa Oportunidades (el programa más grande de combate a la pobreza en México), existen estudios de caso (Quezada, 2011) que muestran el caso de México como nación paradigmática de malas prácticas públicas en materia de atención a los grupos más pobres. Se sabe que en México no existe ningún mecanismo institucional ni legal (no existe Ley reguladora en la materia) que garantice que los más pobres tengan acceso a los programas sociales o para evitar que los beneficios de los programas sociales lleguen a sectores de la población que no lo necesitan. Por ejemplo, 27.3\% del presupuesto de programas sociales en el estado de México, llega a manos del 20\% más rico de la población (CIEPS, 2010:23). Programas Públicos como "Oportunidades" tienen padrones de beneficiarios integrados de manera arbitraria, la selección de los beneficiarios está bajo control de los grupos políticos locales en cada estado de la nación. De esta forma, en las elecciones federales para Presidente de la República en el año 2012 hubo denuncias de irregularidades en el uso de los programas sociales; por parte de autoridades locales en casi todos los estados de México. La falta de normas da origen a la opacidad en el uso de los recursos públicos en México y genera prácticas de política pública sin rendición de cuentas.

El caso de la pobreza patrimonial que afecta el tamaño de la clase media, también ilustra la ausencia de programas públicos de atención a un sector de la población que, históricamente, ha sido el motor del desarrollo de la Nación. A partir de la crisis económica mundial iniciada en el año 2008 una gran parte de la población de clase media en México cayó en insolvencia para el pago de créditos hipotecarios: en el año 2007 el índice de morosidad en el pago de créditos hipotecarios adquiridos en las Sociedades Financieras de Objeto Limitado (que otorgan crédito hipotecario a la clase media en México) era de 4.1\%; índice que creció hasta llegar a 12.8\% en el último trimestre del año 2011 (BANCOMER, 2012:12). También creció la insolvencia en el pago de cuotas en Universidades privadas y en planes de salud privados. El gobierno mexicano no tiene programas de atención a este tipo de problemas que afectan, fundamentalmente, a la clase media.

Algunos países como Estados Unidos, Reino Unido y España han instrumentado programas para apoyar a deudores que enfrentan dificultades para mantenerse al corriente en sus pagos. El gobierno estadounidense, por ejemplo, lanzó un plan para ofrecer apoyo a entre 7 y 9 millones de deudores hipotecarios, que involucra reestructuras de hipotecas para ciertos deudores, aún cuando éstas hayan sido bursatilizadas. El programa contempla en general mejoras en los términos de los créditos, como reducciones del pago mensual mediante la ampliación de plazos y/o reducción de tasas e inclusive quitas de capital bajo ciertas circunstancias.

En el caso de México los programas públicos no atienden, ni han generado programas para apoyar y fortalecer a la clase media. Consideramos relevante que en el seno del 
Congreso de la Unión las fuerzas políticas realicen un pacto político para atender, en los presupuestos de egresos de la Federación, a este sector de la sociedad mexicana. De igual forma, el ejecutivo debe emprender acciones unilaterales de apoyo, desde la Secretaría de Economía y desde la Secretaría de Desarrollo Social Federal, con programas específicos, a la clase media mexicana.

Finalmente, es importante señalar que detrás de la debilidad de la clase media mexicana y del aumento de la pobreza, la ausencia más relevante en la política pública en México es una política salarial activa, que garantice aumentos permanentes de poder de compra. Desde hace más de treinta años el salario mínimo real en México ha perdido poder de compra sistemáticamente. Los salarios mínimos en México son un "semáforo" que indica a las empresas cómo deben aumentar los salarios de sus empleados. De tal forma que la política salarial ha contribuido, fundamentalmente, al aumento de la pobreza y a la caída de la clase media.

\section{ReFERÊNCIA BibliográficA}

AGUILAR, Gutiérrez Genaro ¿Puede el crecimiento eliminar la pobreza? Editor Instituto de Investigaciones Económicas de la Universidad Nacional Autónoma de México, México: 2009.

. Desigualdad y Pobreza en México, ¿son inevitables? Porrúa, México: Porrúa, 2000.

Comparaciones intertemporales de distribuciones del ingreso: México y Brasil. Economía y Tecnología; v. 1, n. 2, p. 26-28, jul./sept. 1998.

BBVA-RESEARCH. Situación Inmobiliaria en México. Análisis Económico, México: Bancomer; Enero 2012.

CALVA, José Luis. Reforma Económica para el Crecimiento Económico Sostenido con Equidad. Economía UNAM, v. 7, n. 21, p. 15-36 UNAM, 2010.

CIEPS. Cuadernos de Investigación \#7. Consejo de Investigación y Evaluación de la Política Social, Gobierno del estado de México, 2010.

DAVIS, Joe C and Huston, John H. The Shrinking Middle-income Class: A Multivariate Analysis. Eastern Economic Journal, v. 18; n. 3, p. 277-285, summer 1992.

ESTEBAN, Joan Maria; GRADÍN, Carlos; RAY, Debraj. An extension ofa measure of polarization, with an application to the income distribution of five OECD countries. Journal of Economic Inequality, v. 5, n. 1, p. 1-19, 2007.

. Extensions of a measure of polarization with an application to the income distribution of five OECD countries. Maxwell School of Citizenship and Public Affairs-Syracuse University. Working Paper, n. 218, p. 1-31, 1999.

ESTEBAN, Joan M.; Ray, Debraj. On the measurement of polarization. Econometrica, v. 62, n. 4, p. 819-852, 1994.

GUILLÉN, Héctor. La contrarrevolución neoliberal, México. Era, 1996.

KUTTNER, Bob. The decline middle-class. Atlantic Montly, n. 252, p. 60-71, 1983.

QUEZADA Limon, Fabiola. El funcionamiento del programa Oportunidades en el municipio de Autlán de Navarro. Tesis de Doctorado en Desarrollo Económico y Sectorial Estratégico, México:UPAEP, 2011.

ROSENTHAL , Neal H. The shrinking middle class: myth or reality? Montly Labour Review, v. 108, n. 3, p. 3-10, march 1985. 
THUROW, Lester. The disappearance of the middle class. New York Times, section F3, p. 3, february 5, 1984.

WOLFSON, Michael C. When inequalities diverge. American Economic Review, v. 84, n. 2; p. 353358. 1994.

WILLIAMSON, John. The Progress of Policy Reform in Latin America. Washington, D. C: IIE, 1990. 